\title{
CONSUMO DE ALCOHOL Y SU RELACIÓN CON LA AUTOPERCEPCIÓN ADOLESCENTE
}

\section{ALCOHOL CONSUMPTION AND ITS RELATIONSHIP WITH THE ADOLESCENT SELF-PERCEPTION}

\author{
Alejandra Calero ${ }^{1}$, Vanina Schmidt ${ }^{1}$ y Lucía Bugallo² \\ ${ }^{1}$ Instituto de Investigaciones, Facultad de Psiología, Universidad de Buenos Aires - CONICET
}

${ }^{2}$ Universidad Nacional del Comahue - CONICET

\section{Abstract}

Foundation. Alcohol consumption in adolescence is a priority public health issue, due to its prevalence in this age group. Additionally, self-perceptions are very important for adolescent development. b) Aims. To study the relationship between the amount and frequency of alcohol consumption and the domains of self-concept and self-esteem during adolescence. c) Methodology. 397 adolescent students of the Autonomous City of Buenos Aires answered a questionnaire about quantity $x$ frequency and HED alcohol consumption and the SelfPerception Profile. d) Results. Self-concept domains of attractive loving and social acceptance were positively associated to alcohol consumption, as well as self-esteem towards the end of adolescence. While academic and behavioral self-concept were associated to alcohol consumption. Differences in results depending on the level of education and sex, even among moderate drinkers and abstainers were found comparing to HED group. e) Conclusion. A positive self-perception in the domains related to the social sphere is associated with increased consumption, while positive self-perceptions in the behavioral and academic domains are associated with lower alcohol consumption. These results are interpreted considering the influence of the social context in the construction of self-concept.

Keywords: adolescence, self-esteem, self-concept, alcohol consumption.

\section{Resumen}

Fundamentación. El consumo de alcohol en la adolescencia representa un problema de salud pública prioritario dada su prevalencia en este grupo etario. Asimismo, las autopercepciones tienen una gran importancia para el desarrollo adolescente. Objetivos. Estudiar la relación entre la cantidad y frecuencia de consumo de alcohol y los dominios del autoconcepto y la autoestima durante la adolescencia. Metodología. Participaron 397 adolescentes escolarizados de la Ciudad Autónoma de Buenos Aires, quienes respondieron el Cuestionario Consumo -Frecuencia- CEEA y el Perfil de Autopercepción. Resultados. Los dominios del autoconcepto atractivo amoroso y aceptación social mostraron estar positivamente asociados al consumo de alcohol, al igual que la autoestima hacia el final de la adolescencia. El autoconcepto académico y la autopercepción positiva sobre el comportamiento mantuvieron una relación negativa. Se encontraron diferencias en los resultados en función del grado de escolaridad y el sexo, también entre abstinentes y consumidores moderados con CEEA. Conclusión. Una autopercepción positiva en dominios referidos a la esfera social se asocia a mayor consumo, mientras que autopercepciones positivas en lo comportamental y académico se asocian a menor consumo de alcohol. Se interpretan estos resultados considerando la influencia del contexto social en la construcción del sí mismo.

Palabras clave: adolescencia, autoconcepto, autoestima, consumo de alcohol. 
Durante la adolescencia la asunción de riesgos está particularmente agudizada en comparación con la adultez, entendiendo por conducta de riesgo aquella que conlleva un daño potencial para sí o terceros (Furby \& Beyth- Marom, 1992). Entre las conductas de riesgo más comunes en los adolescentes de las Américas se encuentra el consumo de alcohol (Monteiro, 2013; OPS: Organización Panamericana de la Salud, 2015). Esta conducta se encuentra naturalizada en la cultura juvenil y se encuentra asociada con una gran cantidad de perjuicios individuales, sociales y económicos (OAD: Observatorio Argentino de Drogas, 2012). Estudios epidemiológicos realizados en Argentina indican que el alcohol es la sustancia más consumida por este grupo etario (OAD, 2012; OAD, 2014).

El consumo de alcohol se ha convertido en un importante problema de salud pública por su ingesta excesiva, riesgosa y cada vez a más temprana edad (Míguez, 2008; OMS: Organización Mundial de la Salud, 2008). Sin embargo, si bien en la adolescencia toda forma de consumo se considera de riesgo, existen ciertas formas de consumo que implican un riesgo mayor sobre todo si es de inicio temprano (OPS, 2015). Una de las formas más riesgosas de consumo de alcohol observado en nuestra población juvenil es el denominado consumo episódico excesivo de alcohol (CEEA), definido como la ingesta de una gran cantidad de alcohol (más de 5 Unidades de Bebida o 60 grs. de alcohol absoluto en sangre) en un lapso breve.

Estudios previos muestran que el efecto del consumo de alcohol puede ser diferencial de acuerdo al patrón de consumo. En estudios longitudinales en donde se estudiaba la relación entre el consumo durante la adolescencia y sus consecuencias en el desarrollo, se encontró que un consumo moderado durante la adolescencia, no sólo no traería aparejado un alto riesgo de consecuencias negativas a largo plazo como un desarrollo neurocognitivo deficiente o una mayor probabilidad de desarrollar conductas problemáticas, entre otras (Chassin, Flora, \& King, 2004; Chassin, Pitts, \& Prost, 2002; Eccles \& Barber, 1999), sino que ayudaría a un mayor ajuste psicológico al terminar la adolescencia, favoreciendo el desarrollo de la autoestima y disminuyendo la probabilidad de experimentar problemas socioemocionales (Chassin et al., 2004; Leifman, Kuhlhon, Allebeck, Andréasson, \& Romelsjö, 1995; Oliva, Parra, \& Sánchez-Queija, 2008). LoS abstinentes mostraban mayores grados de ansiedad, menos de tolerancia al estrés, menores habilidades sociales, menor autoestima y un menor ajuste emocional que los consumidores medios, que como contrapartida presentaban mayores niveles de ajuste conductual. La actitud adolescente conservadora y de evitación de riesgos traería menos problemas conductuales y de salud, pero un desarrollo deficiente en el logro de la identidad personal, la creatividad, la iniciativa, la tolerancia al estrés o las estrategias de afrontamiento (Oliva et al., 2008). Mientras que los consumidores abusivos (aquellos que consumen más de 5 unidades por ocasión), tenían problemas generalizados en los ámbitos académico, psicológico y social, además de mayores probabilidades de desarrollar problemas futuros como depresión, ansiedad o dependencia (Chassin et al., 2002; OPS, 2015; Pilatti, Godoy, Brussino, \& Pautassi, 2013a).

En algunos estudios, la relación entre el autoconcepto y autoestima con el consumo de sustancias no es concluyente (Musitu \& Olaizola, 2003; Naranjo \& González, 2012), y en otros estudios, dicha relación es negativa o positiva o bien depende del tipo de consumo (Oliva et al., 2008). Dolcini y Adler (1994) plantean que a la relación entre las conductas de riesgo y la autoestima podría faltarle claridad por el nivel de generalidad que la autoestima tiene. Desde esta perspectiva, creen que sería recomendable estudiar la relación del consumo de alcohol con los dominios específicos del autoconcepto. De esta manera, tendrían efectos diferenciales distintos aspectos del self sobre el consumo de alcohol (Musitu \& Olaizola, 2003).

Los estudios previos realizados en adolescentes han hallado relaciones positivas entre el autoconcepto físico y el consumo de alcohol (Moreno, Moreno, \& Cervelló, 2009; Musitu, Jiménez, \& Murgui, 2007). También se han encontrado relaciones positivas entre el autoconcepto social (McKay, Sumnall, Cole, \& Percy, 2012; Musitu et al., 2007; Musitu \& Olaizola, 2003; Naranjo \& González, 2012), el dominio del autoconcepto amistad íntima y el consumo de sustancias y relaciones negativas, entre este último, y el autoconcepto sobre el comportamiento (Pastor, Balaguer, \& García-Merita, 2006). En relación al dominio del autoconcepto académico los resultados encontrados en los antecedentes son dispares, mientras que en algunos no se encontró una relación (Pastor et al., 2006) en otros se halló una relación negativa (Gómez, Ortega, \& Ciairano, 2012; Jiménez, Musitu, \& Murgui, 2006; McKay 
et al., 2012; Musitu \& Olaizola, 2003). Por otra parte, algunos estudios plantean que controlando estadísticamente una variable (por ejemplo, el sexo o la edad), la aparente relación estadísticamente significativa entre el autoconcepto social y el consumo de sustancias desaparece (Fuentes, García, Gracia, \& Lila, 2011).

La autoestima es definida como el nivel general de valoración de la propia persona, mientras que el autoconcepto se define como el juicio evaluativo del sí mismo en dominios específicos de la vida (Harter, 1999).

Actualmente, tanto a nivel local como internacional, se plantean nuevos abordajes que apuntan a la prevención del consumo excesivo de alcohol a través de la promoción de actividades que fomenten el desarrollo positivo durante la adolescencia, dentro del cual se encuentra el autoconcepto y autoestima (Parra, Oliva, \& Antolín Suárez, 2009).

El objetivo de este artículo es estudiar la relación entre la cantidad, frecuencia y el CEEA con los dominios del autoconcepto y la autoestima durante la adolescencia.

\section{MÉTODO}

El diseño del estudio es no experimental transversal con alcance descriptivo correlacional (Hernández Sampieri, Fernández-Collado, \& Baptista Lucio, 2010).

\section{Participantes}

La muestra estuvo compuesta por 397 adolescentes que asistían a escuelas secundarias privadas de la Ciudad Autónoma de Buenos Aires. Es una muestra no probabilística por conveniencia (Hernández Sampieri et al., 2010) , es decir, se contactó a instituciones educativas y se procedió a explicar en qué consistía la investigación $y$, finalmente, se trabajó en aquellas que accedieron a participar del estudio.

Todos los adolescentes participaron con consentimiento informado por escrito por parte de sus padres. Se garantizó el anonimato y confidencialidad de los datos.

\section{Instrumentos}

Perfil de Autopercepción. (Facio, Resett, Braude, \& Benedetto, 2006; Harter, 1988): el objetivo de esta escala es evaluar la autoestima global y el autoconcepto (perfil de los juicios evaluativos) a través de distintos dominios específicos del adolescente. Está compuesto por nueve subescalas: Autoestima Global, corresponde a la evaluación global del sí mismo, evalúa en qué medida el adolescente está contento con cómo lleva adelante su vida y con ser como es. A diferencia de la competencia percibida en dominios específicos, se trata de un juicio global de valor global (e.g. “Algunos adolescentes están muy contentos siendo como son, pero otros quisieran ser diferentes de como son"). Atractivo Amoroso, evalúa en qué medida el adolescente se considera atractivo o interesante para aquellas personas con quienes estaría interesado en tener una relación amorosa (e.g. "Algunos adolescentes creen que si se enamoran de alguien esa persona también gustará de ellos, pero a otros les preocupa que si se enamoran de alguien esa persona no guste de ellos"). Apariencia Física, evalúa en qué medida el adolescente está conforme con su apariencia (e.g. "Algunos adolescentes desearían que su cuerpo fuera diferente, pero a otros les gusta su cuerpo tal como es"). Aceptación Social, evalúa en qué medida el adolescente se siente aceptado por sus pares, tiene amigos y cree caerle bien a la gente (e.g. "A algunos adolescentes les resulta difícil caerle bien a los demás, pero a otros les resulta fácil caerle bien a los demás"). Amistad íntima, evalúa la habilidad que el adolescente cree que tiene para hacer amigos íntimos (e.g. "Algunos adolescentes tienen un amigo/a íntimo/a con quien compartir secretos, pero otros no tienen un verdadero amigo/a íntimo/a con quien compartir secretos"). Competencia Deportiva, evalúa cómo percibe el adolescente su habilidad en los deportes (e.g. "Algunos adolescentes creen que son mejores que otros de su edad en los deportes, pero otros adolescentes no creen ser tan buenos en los deportes"). Competencia Escolar, evalúa cómo percibe el adolescente su habilidad en el ámbito académico (e.g. "Algunos adolescentes piensan que son tan inteligentes como otros de su edad, pero otros no están seguros de ser tan inteligentes como otros de su edad"). Buen Comportamiento, evalúa en qué medida está satisfecho con su comportamiento, evita meterse en problemas y cree hacer lo correcto (e.g. "Algunos adolescentes generalmente se meten en problemas por las cosas que hacen, pero otros no se meten en problemas por las cosas que hacen"). Cada ítem 
del Perfil presenta dos afirmaciones opuestas y cuatro opciones de respuesta con puntajes que varían de 1 a 4; donde los puntajes más altos reflejan mayor competencia percibida. La adaptación local cuenta con adecuados indicadores de validez y confiabilidad (Facio et al., 2006). En la muestra con la que se realizó el presente artículo se evaluó la consistencia interna de las nueve subescalas utilizando la estadística alphas de Cronbach. Los alphas obtenidos fueron: apariencia física .88, atractivo amoroso .74, amistad íntima .74, aceptación social .71, buen comportamiento .73, competencia escolar .67, competencia deportiva .84 y autoestima global .75. Los ítems con menor correlación con el puntaje total corregido de su respectiva subescala fueron en todos los casos superiores a .3 a excepción de un ítem de amistad íntima. Al igual que en otros trabajos (Pastor, Balaguer \& García-Merita, 2008) el dominio competencia laboral fue eliminado debido a que la población con la que se trabajo no es laboralmente activa.

Cuestionario sobre consumo y frecuencia de alcohol. Las preguntas que los componen fueron desarrolladas ad hoc y utilizadas en estudios previos (Cremonte, Cherpital, Bosges, Peltzer, \& Santángelo, 2011). Esta modalidad de evaluación de los patrones de consumo ha mostrado adecuados índices de sensibilidad y especificidad. En este cuestionario se evalúa la cantidad (e.g. Cuando toma alguna bebida alcohólica, generalmente ¿cuántos tragos toma en cada ocasión?), frecuencia (e.g. Durante los últimos doce meses ¿qué tan seguido tomó una bebida alcohólica?) y CEEA (e.g. ¿Qué tan seguido tomas 5 o más tragos en una misma ocasión?). En la pregunta sobre cantidad se le brinda al adolescente un cuadro de equivalencias para permitir llevar las distintas bebidas a una misma medida: a más tragos, más alcohol. En las preguntas sobre frecuencia y cantidad los valores más bajos reflejan un menor consumo de alcohol.

\section{Procedimiento}

Se realizó la administración de los instrumentos en forma grupal en el aula durante el horario de clase en la institución educativa, sin profesores presentes y con consentimiento informado por escrito de los padres. El instrumento fue entregado personalmente a los adolescentes en sobre cerrado, quienes debían contestar en forma anónima. A los sujetos se les pedía que marquen el grado de acuerdo con cada una de las opciones de respuesta o bien que respondan el cuestionario. Una vez completado los instrumentos, los sujetos los devolvían a los evaluadores en sobre cerrado.

\section{Análisis de datos}

Para estudiar la relación entre el consumo de alcohol (frecuencia, cantidad y CEEA) y el perfil de autopercepción, se utilizó la prueba no paramétrica Rho de Spearman. Las correlaciones se realizaron por sexo y por año al que asistía el adolescente. Se utilizó la corrección de Bonferroni para ajustar el p valor. Para estudiar la relación entre el tipo de consumo: abstinentes, moderados (de una 1 a 4 unidades de alcohol por ocasión) y CEEA (más de 5 unidades de bebidas por ocasión) y el Perfil de autopercepción se utilizó la prueba no paramétrica Kruskall Wallis. Para efectuar las comparación pos hoc se utilizó la prueba U de Mann Whitney con corrección de Bonferroni para ajustar el p valor.

Se utilizaron estadísticos no paramétricos debido a la falta de normalidad de las variables incluidas en el estudio.

Se utilizó el paquete estadísticos SPSS versión 18.

\section{RESULTADOS}

La muestra estuvo compuesta por 262 mujeres (\%66) y 135 varones con una media de edad de 15.15 (DE= 1.863). Del total 135 (34\%) con una media de edad de 13.12 ( $D E=.387 ;$ Min=12; Max=14) asistían a $1^{\circ}$ año, 139 (35\%) con una media de edad de 15.17 (DE=.398; Min=15; Max=16) asistían a $3^{\circ}$ año y $123(31 \%)$ con una media de edad de 17.43 ( $D E=.513 ; \operatorname{Min}=17 ; \operatorname{Max}=19)$ a $5^{\circ}$ año de la escuela secundaria.

Respecto de la correlación entre las variables de consumo de alcohol con la autoestima y los dominios específicos del autoconcepto se obtuvieron, en el caso de las mujeres, correlaciones positivas significativas entre frecuencia y atractivo amoroso (Rho =.224; $p<.001$ ). También se encontraron correlaciones positivas significativas entre cantidad y atractivo amoroso ( $R$ ho = .212; $p=.036$ ). Pero se halló una asociación negativa entre cantidad y buen comportamiento ( $R h o=-.179 ; p=.048$ ). En el caso de los varones, se obtuvieron correlaciones significativas entre frecuencia y atractivo amoroso (Rho 
$=.317 ; p<.001)$ y entre cantidad de consumo de alcohol y atractivo amoroso (Rho $=.269 ; p=.024)$ (ver Tabla 1$)$.

Tabla 1. Correlaciones entre autoconcepto y autoestima con frecuencia y cantidad por sexo

\begin{tabular}{|c|c|c|}
\hline \multicolumn{3}{|l|}{ Mujer } \\
\hline & $F A(n=260)$ & CA $(n=258)$ \\
\hline & Rho. & Rho. \\
\hline ATE & -.053 & -.068 \\
\hline AAF & -.133 & -.149 \\
\hline AAA & $.224^{\star *}$ & $.212^{* *}$ \\
\hline AAI & .041 & .017 \\
\hline AAS & .006 & -.024 \\
\hline AM & -.126 & $-.179^{*}$ \\
\hline ACM & -.073 & -.051 \\
\hline$A C D$ & -.088 & -.092 \\
\hline \multicolumn{3}{|l|}{ Varón } \\
\hline & $F A(n=397)$ & CA $(n=392)$ \\
\hline & Rho. & Rho. \\
\hline ATE & .087 & .040 \\
\hline AAF & .145 & .153 \\
\hline AAA & $.317^{* *}$ & $.269^{*}$ \\
\hline AAI & -.065 & -.098 \\
\hline AAS & .088 & .028 \\
\hline AM & -.196 & -.234 \\
\hline $\mathrm{ACM}$ & -.132 & -.218 \\
\hline$A C D$ & -.037 & .045 \\
\hline
\end{tabular}

Nota: Rho: Rho de Spearman. Sig: Significación. FA: Frecuencia consumo alcohol. CA: Cantidad consumo de alcohol. ATE: Autoestima. Dominios del Autoconcepto= AAF: Apariencia física. AAA: Atractivo amoroso. AAl: Amistad íntima. AAS: Aceptación social. AM: Buen comportamiento. ACM: Competencia escolar. ACD: Competencia deportiva. ${ }^{*} \mathrm{p}<.05 .{ }^{*} \mathrm{p}<.001$ Ajustado por corrección de Bonferroni.

Al realizar correlaciones entre el autoconcepto y autoestima y las variables de consumo de alcohol por año al que asistía el adolescente, no se obtuvieron correlaciones significativas en primer año. En tercer año se obtuvo una correlación significativa positiva entre aceptación social y frecuencia (Rho =.270; $p=.012$ ). También se hallaron correlaciones significativas negativas entre buen comportamiento y frecuencia (Rho $=.-310 ; p<.001)$ y cantidad $(R h o=-.327 ; p<.001)$. En quinto año se encontraron correlaciones significativas positivas entre autoestima global y frecuencia de consumo (Rho $=.259 ; p=.048$ ) y entre atractivo físico y cantidad de alcohol (Rho =.259; $p=.024)$. También se encontraron correlaciones significativas negativas entre buen comportamiento y cantidad (Rho =-.280; $p=.024$ ) y entre competencia escolar y cantidad (Rho $=-.302 p=.012$ ) (ver Tabla 2).
Tabla 2. Correlaciones entre autoconcepto y autoestima con frecuencia y cantidad de alcohol por año al que asiste el adolescente

\begin{tabular}{|c|c|c|}
\hline $\begin{array}{l}\text { Primer año del } \\
\text { secundario }\end{array}$ & $F A(n=133)$ & $C A(n=131)$ \\
\hline & Rho. & Rho. \\
\hline ATE & -.156 & -.065 \\
\hline AAF & -.127 & -.070 \\
\hline AAA & .133 & .030 \\
\hline AAI & -.021 & -.011 \\
\hline AAS & -.025 & .070 \\
\hline AM & -.092 & -.153 \\
\hline ACM & -.016 & -.082 \\
\hline ACD & .024 & .100 \\
\hline \multirow[t]{2}{*}{$\begin{array}{l}\text { Tercer año del } \\
\text { secundario }\end{array}$} & $\mathrm{FA}(\mathrm{n}=139)$ & $C A(n=138)$ \\
\hline & Rho. & Rho. \\
\hline ATE & .083 & .023 \\
\hline AAF & .053 & -.029 \\
\hline AAA & .205 & .197 \\
\hline AAI & .062 & .008 \\
\hline AAS & $.270^{*}$ & .161 \\
\hline AM & $-.310^{* *}$ & $-.327^{* *}$ \\
\hline ACM & -.216 & -.182 \\
\hline$A C D$ & .019 & -.003 \\
\hline \multirow[t]{2}{*}{$\begin{array}{l}\text { Quinto año del } \\
\text { secundario }\end{array}$} & $\mathrm{FA}(\mathrm{n}=397)$ & $C A(n=392)$ \\
\hline & Rho. & Rho. \\
\hline ATE & $.259^{*}$ & .153 \\
\hline AAF & .255 & $.277^{*}$ \\
\hline AAA & .154 & .115 \\
\hline AAI & -.086 & -.059 \\
\hline AAS & .120 & .102 \\
\hline AM & -.199 & $-.280^{*}$ \\
\hline ACM & -.256 & $-.302^{*}$ \\
\hline ACD & .041 & .072 \\
\hline
\end{tabular}

Nota: Rho: Rho de Spearman. Sig: Significación. FA: Frecuencia consumo alcohol. CA: Cantidad consumo de alcohol. ATE: Autoestima. Dominios del Autoconcepto= AAF: Apariencia física. AAA: Atractivo amoroso. AAl: Amistad íntima. AAS: Aceptación social. AM: Buen comportamiento. ACM: Competencia escolar. ACD: Competencia deportiva. ${ }^{*} p<.05 .{ }^{* \star} p<.001$ Ajustado por corrección de Bonferroni

Al realizar una comparación entre el grupo de abstinentes, los consumidores moderados y CEEA se encontró una diferencia significativa en apariencia física, atractivo amoroso y buen comportamiento (ver Tabla 3).

Se procedió a realizar comparaciones por pares para analizar dónde se encontraba la diferencia detectada. El análisis post hoc indica, luego de corregir el p valor por el índice de Bonferroni, que entre los abstinentes y los CEEA existe una diferencia significativa en atractivo amoroso siendo mayor en los CEEA y en buen comportamiento siendo mayor en los abstinentes (AAA: 
$U=3081,50 ; z=-4,295 ; p<.001 ;$ AM: $U=2965,00 ; z=-$ 4,576; $p<.001)$. También, se halló una diferencia significativa entre los consumidores moderados y CEEA en el dominio buen comportamiento siendo mayor en los consumidores moderados (AM: $U=3729.50 ; z=-4.541 ; p$ $<$.001). Finalmente, no se hallaron diferencias significativas entre los abstinentes y los consumidores moderados (ver Tabla 4).

Tabla 3. Comparación del autoconcepto y autoestima en abstinentes, moderados y CEEA

\begin{tabular}{rrrlll}
\hline & $\begin{array}{c}\text { Modern. } \\
(\mathrm{n}=178)\end{array}$ & $\begin{array}{c}\text { CEEA } \\
(\mathrm{n}=67)\end{array}$ & $\mathrm{U}$ & $\mathrm{Z}$ & Sig. \\
ATE & 121.10 & 128.06 & 5624.00 & -.690 & .490 \\
AAF & 116.14 & 141.23 & 4741.50 & -2.479 & .013 \\
AAA & 116.29 & 140.81 & 4769.50 & -2.424 & .015 \\
AAI & 129.66 & 105.31 & 4778.00 & -2.413 & .016 \\
AAS & 124.22 & 119.75 & 5745.00 & -.445 & .656 \\
AM & 135.55 & 89.66 & 3729.50 & -4.541 & $.000^{*}$ \\
ACM & 130.65 & 102.66 & 4600.50 & -2.770 & .006 \\
ACD & 122.20 & 125.13 & 5820.00 & -.290 & .772 \\
\hline
\end{tabular}

Nota: U: U de Mann Whitney. gl: Grados de libertad.. Sig: Significación. FA Frecuencia consumo alcohol. CA: Cantidad consumo de alcohol. ATE: Autoestima. Dominios del Autoconcepto= AAF: Apariencia física. AAA: Atractivo amoroso. AAl: Amistad íntima. AAS: Aceptación social. AM: Buen comportamiento. ACM: Competencia escolar. ACD: Competencia deporłba.Moder.: Modegados* $p<.05$ Ajustg. S.do por Bonferroni

\begin{tabular}{|c|c|c|c|c|c|}
\hline ATE & 197.53 & 191.45 & 201.87 & .496 & .780 \\
\hline AAF & 207.29 & 177.58 & 217.58 & 8.706 & $.013 *$ \\
\hline AAA & 170.74 & 198.98 & 239.82 & \multirow{2}{*}{ 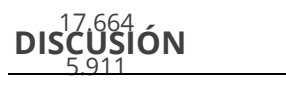 } & $.000 * *$ \\
\hline AAI & 193.27 & 207.42 & 168.67 & & .052 \\
\hline AAS & 194.82 & 197.81 & 190.83 & \multirow{4}{*}{\multicolumn{2}{|c|}{ 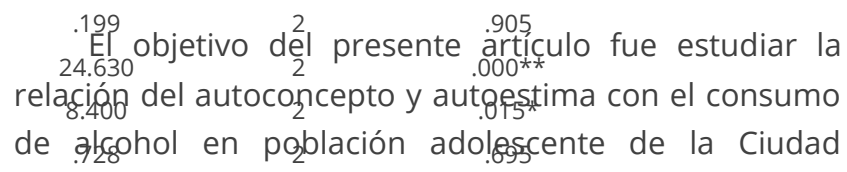 }} \\
\hline AM & 211.68 & 205.50 & 133.92 & & \\
\hline ACM & 202.98 & 203.01 & 159.37 & & \\
\hline$A C D$ & 201.49 & 190.77 & 195.11 & & \\
\hline
\end{tabular}

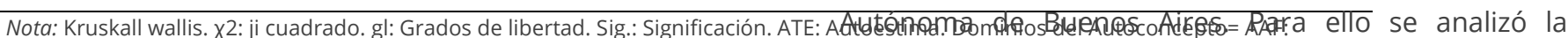

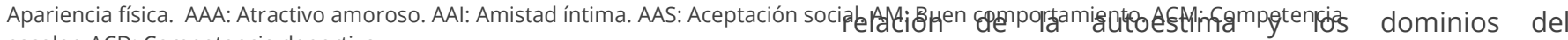
escolar. ACD: Competencia deportiva.

$* p<.05 . * * p<.001$. Ajustado por Bonferroni.

autoconcepto con la frecuencia, cantidad y patrón de consumo de alcohol.

Tabla 4. Comparaciones por pares entre abstinentes, consumidores moderados y tipo CEEA.

\begin{tabular}{|c|c|c|c|c|c|}
\hline \multicolumn{6}{|c|}{ Rango Promedio } \\
\hline & $\begin{array}{l}\text { Abstinent } \\
\text { es }(n=145)\end{array}$ & $\begin{array}{c}\text { CEEA } \\
(n=67)\end{array}$ & $U$ & $\mathrm{Z}$ & Sig. \\
\hline ATE & 105,89 & 107,81 & 4769.50 & -.213 & .831 \\
\hline AAF & 104,72 & 110.35 & 4599.50 & -.623 & .533 \\
\hline AAA & 94,25 & 133.01 & 3081.50 & -4.295 & $.000^{*}$ \\
\hline AAl & 110,72 & 97.36 & 4245.00 & -1.483 & .138 \\
\hline AAS & 107,16 & 103,98 & 4762.50 & -.231 & .817 \\
\hline $\mathrm{AM}$ & 119,55 & 78.25 & 2965.00 & -4.576 & $.000 *$ \\
\hline ACM & 113,80 & 90.71 & 3799.50 & -2.560 & .010 \\
\hline$A C D$ & 107,67 & 103,98 & 4688.50 & -.408 & .683 \\
\hline \multicolumn{6}{|c|}{ Rango Promedio } \\
\hline & $\begin{array}{l}\text { Abstinent } \\
\text { es }(n=145)\end{array}$ & $\begin{array}{l}\text { Modern. } \\
(n=178)\end{array}$ & $U$ & Z & Sig. \\
\hline ATE & 164.63 & 159.85 & 12523.00 & -.460 & .646 \\
\hline AAF & 175.57 & 150.95 & 10937.50 & -2.364 & .018 \\
\hline AAA & 149.49 & 172.19 & 11091.50 & -2.182 & .029 \\
\hline AAI & 155.54 & 167.26 & 11969.00 & -1.128 & .259 \\
\hline AAS & 160.66 & 163.09 & 12711.00 & -.234 & .815 \\
\hline AM & 165.12 & 159.46 & 12452.00 & -.546 & .585 \\
\hline ACM & 162.18 & 161.85 & 12878.50 & -.032 & .975 \\
\hline$A C D$ & 166.82 & 158.07 & 12206.00 & -.840 & .401 \\
\hline \multicolumn{6}{|c|}{ Rango Promedio } \\
\hline
\end{tabular}

Los datos hallados deben ser interpretados en el marco del contexto social y cultural del cual emergen. En nuestro contexto, el consumo de alcohol se encuentra naturalizado y el consumo ocasional de grandes cantidades en escenarios nocturnos y con grupos de pares genera un estereotipo respecto de lo que es "esperable" y, hasta, "ideal" entre los adolescentes (Míguez, 2008).

Al comparar los grupos abstinentes, de consumo moderado y de CEEA se encontró que existían diferencias en la forma en que los adolescentes se percibían en los dominios atractivo amoroso y buen comportamiento entre el grupo de abstinentes, quienes puntuaron más alto en este último y más bajo en el primer dominio, y los CEEA. También se halló esta diferencia en el dominio buen comportamiento entre los consumidores moderados y CEEA, siendo estos últimos los que reportaron menores puntajes en este dominio. Asimismo, no se hallaron diferencias significativas entre los abstinentes y los consumidores moderados. Estos hallazgos no coinciden con las conclusiones alcanzadas en investigaciones previas en donde se había encontrado 
un mayor ajuste psicológico reflejado en un mayor nivel de autoestima en los adolescentes que consumían de forma moderada versus abstinentes y CEEA (Chassin et al., 2004; Leifman et al., 1995; Oliva et al., 2008).

Aquellos que consumen intentan acercarse a este estereotipo para ser parte del grupo de pares y alejarse de la inmadurez propia de la infancia (Oliva et al., 2008). Este hecho puede hacer que se perciban como más atractivos a nivel amoroso, asociación que se observó repetidamente a lo largo de los análisis realizados, ya que cuentan con un mayor nivel de aceptación social. En trabajos previos se habían observado relaciones positivas halladas en relación al atractivo físico con el consumo de alcohol (Moreno et al., 2009; Musitu et al., 2007). Desde los medios se brinda una información contradictoria en donde se plantea que el consumo de alcohol es negativo al mismo tiempo que se bombardea con publicidades que resaltan el atractivo de aquellos jóvenes que participan como actores, de modo tal que ocurre una sobrevaloración de la sexualidad como forma de vincularse que se ve relacionada con el consumo de alcohol.

La relación positiva encontrada entre la frecuencia del consumo de alcohol con el dominio aceptación social en los alumnos de tercer año coincide con los resultados hallados en trabajos previos que indican una relación positiva el dominio amistad íntima (Pastor et al., 2006) y el dominio autoconcepto social con el consumo de alcohol (McKay et al.,2012; Musitu et al., 2007; Musitu \& Olaizola, 2003; Naranjo \& González, 2012); esta relación es coherente con trabajos que muestran a partir del análisis del entorno social que la tenencia de amigos que toman alcohol regularmente o que consumen drogas ilícitas era claramente un factor de riesgo (Pilatti, Godoy, Brussino, y Pautassi, 2013b). De todas formas cabe preguntarse como interrogante para continuar explorando en futuras investigaciones sobre la asociación con la aceptación social, variable que mostró ser muy importante en estudios previos, y que en el presente estudio se observó sólo en los adolescentes de tercer año del secundario.

Las relaciones halladas con el dominio de autoconcepto buen comportamiento indican, en todos los casos, que aquellos adolescentes con una mejor autopercepción en este sentido consumían alcohol con menos frecuencia y menos cantidad. Nuevamente, esta asociación, también se reitera en los análisis realizados. Lo cual además de coincidir con investigaciones previas (Pastor et al., 2006) es intuitivamente coherente, más aún si se tiene en cuenta que los adolescentes que no consumen se alejan de este estereotipo del adolescente que consume alcohol de forma desmedida. Cabe destacar que al realizar el análisis por sexo, sólo en las mujeres se observó esta asociación, lo cual podría deberse a que la presión social sobre la mujer y sobre lo que se considera un comportamiento adecuado es mayor que en el caso de los varones.

Asimismo, sólo en los alumnos de quinto año se halló la relación positiva entre la autoestima y la frecuencia y cantidad de consumo de alcohol pero esta relación mostró no ser contundente. Considerando la importancia que tiene el autoestima como un nivel de evaluación del sí mismo que se encuentra en un estadio superior a los dominios específicos del autoconcepto estos resultados muestran la importancia que el consumo de alcohol tiene en esta franja etaria.

Cuando se estudió esta misma relación por el año escolar al que asistía el adolescente, no se obtuvieron correlaciones significativas en primer año. Esto puede deberse al bajo índice de consumo de alcohol que reportaron a esta edad en comparación con los adolescentes que asistían a tercero y quinto año del secundario. Es probable que las oportunidades sociales de consumir alcohol sean más escasas que la de los adolescentes de mayor edad, ya que a esta edad aún no realizan tantas salidas nocturnas. Esta suposición podría complementarse a futuro con un estudio cualitativo a partir de entrevistas con los propios adolescentes sobre sus actividades.

Finalmente, como ya se expuso en la bibliografía, los resultados en relación al autoconcepto académico son dispares (Gómez et al., 2012; Jiménez et al., 2006; McKay et al., 2012; Musitu \& Olaizola, 2003; Pastor et al., 2006). La relación entre el dominio del autoconcepto competencia escolar y la cantidad de alcohol en los alumnos de quinto año podría indicar que el poseer una buena autopercepción en este sentido funciona como una forma de factor protector para la cantidad de alcohol que el adolescente consume o el patrón de consumo o que la cantidad que consumo afecta efectivamente el rendimiento (ver Schmidt, Messoulam, \& Molina, 2008). 
Probablemente no sea la ingesta de la sustancia lo que produce una autopercepción de competencias más elevadas en el dominio social o de apariencia física y/o más baja en el dominio de competencia buen comportamiento. El consumo puede ser visto como una práctica socialmente valorada -además de socialmente muy tolerada- por lo que podría explicar las autopercepciones tan positivas en ciertos dominios y hasta una autoestima más elevada hacia los 18 años, momento de importante cambio en la vida adolescente. Recordemos además, que se ha creado en la sociedad un estereotipo sensacionalista e inexacto del adolescente que lo presenta como problemático $y$, entre sus conductas de riesgo, como alguien que consume alcohol de manera excesiva.

El hecho de que el grupo de CEEA presente resultados tan positivos merece un esfuerzo interpretativo cuidadoso. Basados en resultados previos (Chassin et al., 2004; Leifman et al., 1995; Oliva et al., 2008), era esperable que el grupo de consumidores moderados obtenga los mejores resultados en términos de autoconcepto y autoestima. Sin embargo, en un informe reciente de la OPS (2015), el registro de un aumento del $300 \%$ para mujeres y un aumento del $50 \%$ para varones en la prevalencia de CEEA en las Américas, pueden estar indicando un cambio generacional de "ideales" respecto del consumo de alcohol. Es decir, el consumo de alcohol "ideal" para un adolescente está lejos de lo que el mundo adulto y sus estándares académicos consideran excesivo o moderado. Tal vez hoy, para un adolescente, el consumo excesivo facilita unas percepciones sobre el sí mismo positivas, a pesar de las evidencias de un sinnúmero de consecuencias negativas perfectamente bien establecidas respecto del CEEA. Y de allí que hoy estemos observando que es el CEEA, y no el consumo moderado, el patrón que se asocia a unas autopercepciones más positivas.

Este estudio no está exento de limitaciones. En primer lugar, se ha trabajado con una muestra no probabilística $y$, por lo tanto, no es una muestra representativa. Lamentablemente una muestra aleatoria para este tipo de población es prácticamente imposible. Por otra parte, existen limitaciones geográficas: sólo se trabajó con adolescentes de la Ciudad de Buenos Aires. Por último, sólo se trabajó con adolescentes escolarizados y de clase media. Los adolescentes que participaron del estudio concurren a escuelas privadas, que implican el pago de una matrícula, replicar este estudio con adolescentes que concurren a instituciones educativas públicas o que no se encuentran escolarizados permitiría realizar una generalización de los resultados.

Más allá de la dirección de las relaciones, este estudio invita a pensar nuevas alternativas de intervención en instituciones educativas de nivel medio. De replicarse estos resultados, las estrategias preventivas en población adolescente deberían ir más allá de la indicación de consecuencias negativas, considerando que los grupos que consumen de manera riesgosa son los que perciben mejores competencias (aceptación social, atractivo amoroso, etc.), las cuales son muy valoradas socialmente. El camino, en términos de promoción de la salud, implica trabajar sobre la desnaturalización y la des-idealización del consumo excesivo haciendo foco en estrategias de autocuidado y cuidado del otro, y en la promoción de competencias que permiten desarrollar una vida comprometida y con sentido (Seligman, 2011). Finalmente, no es posible cambiar el comportamiento de los jóvenes respecto del consumo sin modificar el de los adultos (Monteiro, 2013). Los adolescentes son el espejo en el que los adultos podemos mirarnos.

Como posibles líneas futuras que surgen de esta investigación se plantea la posibilidad de replicar el estudio con una muestra de adolescentes más heterogénea en relación a las variables sociodemográficas y socioeconómicas; como así también la inclusión de variables que pueden resultar importantes a la hora de interpretar las variables como las vinculadas a la familia del adolescente.

\section{Agradecimientos}

Esta investigación fue financiada por el Consejo Nacional de Investigaciones Científicas y Técnicas (CONICET; Res. No Res. No 003759/13).

\section{REFERENCIAS}

Cremonte, M., Cherpital, C., Bosges, G., Peltzer, R., \& Santángelo, P. (2011). Drinking patterns and DSM-IV alcohol use disorders' criteria in Argentinean Emergency Department patients. Journal of Drug Addiction, Education and Eradication, 6, 15-30.

Chassin, L., Pitts, S. C., \& Prost, J. (2002). Drinking Trajectories From Adolescence to Emerging Adulthood in a High-Risk Sample: 
Predictors and Substance Abuse Outcomes. Journal of Consulting and Clinical Psychology, 70(1), 67-78. DOI: 10.1037/0022$\underline{006 \times .70 .1 .67}$

Chassin, L., Flora, D. B., \& King, K. M. (2004). Trajectories of Alcohol and Drug Use and Dependence From Adolescence to Adulthood: The Effects of Familial Alcoholism and Personality. Journal of Abnormal Psychology, 113(4), 483-498. DOI: 10.1037/0021-843X.113.4.483

Dirección Nacional de Salud Mental y Adicciones (DNSMA, 2012). Lineamientos para la atención del consumo episódico excesivo de alcohol en adolescentes. Versión online disponible en Julio de 2015: http://www.msal.gov.ar/images/stories/ministerio/adolescencia/20 12-consumo-alcohol.pdf

Dolcini, M. M., \& Adler, N. E. (1994). Perceived competencies, peer group affiliation, and risk behavior among early adolescents. Health Psychology, 13(6), 496. DOI: 10.1037/0278-6133.13.6.496

Eccles, J. S., \& Barber, B. L. (1999). Student Council, Volunteering, Basketball, or Marching Band: What Kind of Extracurricular Involvement Matters? Journal of Adolescent Research, 14(1), 10-43. DOI: $10.1177 / 0743558499141003$

Facio, A., Resett, S., Braude, M., \& Benedetto, N. (2006). El Perfil de Autopercepción de Harter para Adolescentes en jóvenes de Paraná, Río Gallegos y Buenos Aires. Investigación Psicológica, 11(3), 7-25.

Fuentes, M. C., García, F., Gracia, E., \& Lila, M. (2011). Autoconcepto y consumo de sustancias en la adolescencia. Adicciones, 23, 237-248.

Furby, L., \& Beyth- Marom, R. (1992). Risk taking in adolescence: A decision- making perspective. Developmental Review, 12, 1-44. DOI: 10.1016/0273-2297(92)90002-1

Gómez, N., Ortega, E., \& Ciairano, S. (2012). Relación entre el uso de alcohol y condiciones académicas en el adolescente, comparación entre Bolivia, Italia y los Países Bajos. Revista de Investigación Psicológica, 8, 37-55.

Harter, S. (1988). Manual for the Self-Perception Profile for Adolescents. Denver: University of Denver.

Harter, S. (1999). The construction of the self. New York: Guilford.

Hernández Sampieri, R., Fernández-Collado, C., \& Baptista Lucio, P. (2010). Metodología de la investigación. (5ta ed.). México: Mc Graw Hill.

Jiménez, T.I., Musitu, G., \& Murgui, S. (2006). Funcionamiento y comunicación familiar y consumo de sustancias en la adolescencia: el rol mediador del apoyo social. Revista de Psicología Social, 21(1), 21-34.

Leifman, H., Kuhlhorn, E., Allebeck, P., Andréasson, S., \& Romelsjö, A. (1995). Abstinence in late adolescence - Antecedents to and covariates of a sober lifestyle and its consequences. Social Science \& Medicine, 41(1), 113-12. DOI: 10.1016/0277-9536(94)00298-8

McKay, M. T., Sumnall, H. R., Cole, J. C., \& Percy, A. (2012). Self-esteem and self-efficacy: Associations with alcohol consumption in a sample of adolescents in Northern Ireland. Drugs: education, prevention and policy, 19(1), 72-80. DOI: 10.3109/09687637.2011.579585

Míguez, H. (2008). Alcohol y sociedad "líquida". Revista Crepúsculo, 6, 5-10.

Ministerio de Salud (2011). Algunos Datos Sobre el Consumo de Alcohol en Argentina Año 2011. Versión online disponible en Abril de 2015: http://www.msal.gov.ar/saludmental/images/stories/infoequipos/pdf/4-algunos-datos-sobre-el-consumo-de-alcohol.pd

Monteiro, M. (2013). Alcohol y Salud Pública en América Latina: ¿cómo impedir un desastre sanitario? Adicciones, 25, (2), 99-105. Version online:

http://redalyc.org.www.redalyc.org/articulo.oa?id=289126458003

Moreno, J., Moreno, R., \& Cervelló, E. (2009). Relación del autoconcepto físico con las conductas de consumo de alcohol y tabaco en adolescentes. Adicciones, 21(2), 147-154.

Musitu, G., Jiménez, T. I., \& Murgui, S. (2007). Funcionamiento familiar, autoestima y consumo de sustancias en adolescentes: un modelo de mediación. Salud Pública de México, 49(1), 3-10.

Musitu, G., \& Olaizola, J. H. (2003). El rol del autoestima en el consumo moderado de drogas durante la adolescencia. Revista Internacional de Ciencias Sociales y Humanidades, 13(1), 285-306. Versión online: http://www.uv.es/ lisis/gonzalo/14rol-autoest.pdf

Naranjo, C. R., \& González, A. C. (2012). Autoestima en la adolescencia: análisis y estrategias de intervención. International Journal of Psychology and Psychological Therapy, 12(3), 389-403.

Oliva, A., Parra, A., \& Sánchez-Queija, I. (2008). Consumo de sustancias durante la adolescencia: trayectorias evolutivas y consecuencias para el ajuste psicológico. International Journal of Clinical and Health Psychology, 8(1), 153-169. Versión online: http://www.redalyc.org/articulo.oa?id=33780111

Observatorio Argentino de Drogas (OAD, 2012). Quinta Encuesta Nacional a Estudiantes de Enseñanza Media 2011.Versión online disponible en Mayo de 2015: http://portal.educacion.gov.ar/files/2013/10/adiccion_quinta_encu esta.pdf

Observatorio Argentino de Drogas (OAD, 2014). VI Estudio Nacional sobre Consumo de Sustancias Psicoactivas en Estudiantes de Enseñanza Media 2014. Boletín nro. 1: Patrones y Magnitud del Consumo: diagnóstico a nivel país. Buenos Aires: SEDRONAR.

Organización Mundial de la Salud (OMS, 2008). Informe sobre la salud en el mundo 2008. La atención primaria de la salud. Versión online disponible en Diciembre de 2014: http://www.who.int/whr/2008/es/

Organización Panamericana de la Salud (OPS, 2010) El alcohol: un producto de consumo no ordinario. Investigación y políticas públicas, Segunda edición. Versión online disponible en Diciembre de 2014: http://www2.paho.org/hq/dmdocuments/alcoholpoliticas.pdf

Organización Panamericana de la Salud (OPS, 2015). Regional Status Report on Alcohol and Health in the Americas. Washington D.C.: WHO. 
Parra, A., Oliva, A., \& Antolín Suárez, L. (2009). Los programas extraescolares como recurso para fomentar el desarrollo positivo adolescente. Papeles del Psicólogo, 30(3), 3-13. Versión online: http://www.redalyc.org/articulo.oa?id=7781179001

Pastor, Y., Balaguer, I., y García-Merita, M. L. (2008). Testing a proposed model on the relationship between self-concept and a healthy lifestyle in adolescence. International Journal of Psychology Research, 3, 109-128.

Pastor, Y., Balaguer, I., \& García-Merita, M. L. (2006). Relaciones entre el autoconcepto y el estilo de vida saludable en la adolescencia media: un modelo exploratorio. Psicothema, 18(1), 18-24.

Pilatti, A., Godoy, J. C., Brussino, S., y Pautassi, R. (2013a). Underage drinking: prevalence and risk factors associated to drinking experiences among Argentinean children. Alcohol, 47(4), 323-331. DOI: 10.1016/j.alcohol.2013.02.00.

Pilatti, A., Godoy, J.C., Brussino, S.A., \& Pautassi, R.M. (2013b). Patterns of substance use among Argentinean adolescents and analysis of the effect of age at first alcohol use on substance use behaviors, Addictive Behaviors, 38, 2847-2850. DOI: $\underline{10.1016 / j . a d d b e h .2013 .08 .007}$

Seligman, M. E. P. (2011). Flourish: A New Understanding of Happiness and Well-Being - and How To Achieve Them. London: Nicholas Brealey Publishing.

Schmidt, V., Messoulam, N., \& Molina, F. (2008). Autoconcepto académico en adolescentes de escuelas medias: presentación de un instrumento para su evaluación. Revista Iberoamericana de Diagnóstico y Evaluación Psicológica, 25(1), 81-106. 